assessed the age of onset in relation to the presence of autoantibodies. The association with characteristics of the anti-citrullinated protein antibodies (ACPA)-response was also explored.

Objectives: 1) determine the association between age of RA-onset and the presence of ACPA, rheumatoid factor (RF) and anti-carbamylated protein (antiCarP) antibodies, 2) study if age of onset was associated with characteristics of the ACPA-response, 3 ) substantiate previously reported associations between age of onset and clinical characteristics.

Methods: 3,321 1987-positive RA-patients included in the Leiden-EAC, BARFOT, ESPOIR, Umeå and Lund cohorts were studied at presentation on age of onset and the presence of ACPA, RF and anti-CarP antibodies. Logistic regression analyses were performed; effect sizes were summarized in inverseweighted meta-analyses. Within ACPA-positive RA, ACPA-level was studied in all cohorts; ACPA-isotypes, ACPA-fine-specificity and ACPA-avidity index and clinical characteristics were studied in the Leiden-EAC.

Results: From the age of fifty onwards, the proportion of ACPA-negative RApatients increased in Dutch, Swedish and French cohorts. Similar observations were done for RF and anti-CarP. The composition of the ACPA-response did not change with increasing age of onset with respect to titer, isotype distribution, fine specificity and avidity index. With increasing age of onset RA-patients smoked less often, had higher acute phase reactants and more often a sub(acute) symptom onset.

Conclusions: Data of five cohorts revealed that with higher age of onset ACPAnegative RA is more frequent than ACPA-positive RA, while characteristics of ACPA-positive RA as judged by the composition of the ACPA-response appeared not age-dependent. Although more biologic studies are needed to characterize the pathogenesis of ACPA-negative polyarthritis at older age, the present data can promote personalized treatment decisions in ACPA-negative patients in daily practice.

Disclosure of Interest: None declared

DOI: 10.1136/annrheumdis-2017-eular.3781

\section{AB0013 IS THERE IMMUNE DISREGULATION IN NON-SJÖGREN SICCA SYNDROME? A STUDY OF BLOOD LYMPHOCYTE SUBPOPULATIONS}

F. Barcelos ${ }^{1,2,3}$, C. Martins ${ }^{3}$, G. Nunes ${ }^{3}$, T. Lopes ${ }^{3}$, J. Vaz Patto ${ }^{1}$, J. Amaral ${ }^{4}$, J.C. Branco ${ }^{3}$, L.M. Borrego ${ }^{3}$. ${ }^{1}$ Instituto Português Reumatologia; ${ }^{2}$ Hospital CUF Descobertas; ${ }^{3}$ Nova Medical School; ${ }^{4}$ Faculdade Med Dentária, UL, Lisboa, Portugal

Background: A large number of patients with sicca syndrome not fulfilling Sjögren's syndrome (SS) classification criteria, present manifestations of autoimmunity, like arthritis, Raynaud's, rash or hematologic disturbances, and have anti-nuclear antibodies, lacking however more specific antibodies. The designation Undifferentiated Connective Tissue Disease was coined to refer to those patients, and some will eventually progress to a definite disease, of which SS would be a likely candidate. Immune cell disturbances could be progression markerr, since diseases like pSS have distinct lymphocyte profiles.

Objectives: We aim to study the circulating lymphocyte subsets in non-Sjögren sicca patients (n-SS), and compare them with pSS and healthy controls.

Methods: We included 65 n-SS patients, 53 pSS patients (2002 AECG criteria) and 22 healthy controls. Lymphocyte subsets were characterized by flow cytometry, including follicular and regulatory $\mathrm{T}$ cells and naïve, mature, memory, plasmablasts and regulatory B cells. Statistical analysis was performed with GraphPad, and significance was considered for $\mathrm{p}<0.05$

Results: Comparing to controls, n-SS patients had lower counts of $\mathrm{T}$ cells $(\mathrm{p}=0.016)$, with lower CD4 $(\mathrm{p}=0.0028)$, however that difference was not as pronounced as between SS and controls. n-SS patients had higher percentages of CD4 ( $p=0.0005)$ and lower CD8 percentages $(p=0.0009)$ than pSS. Additionally, there was a decrease in absolute counts of Tregs $(p=0.0028)$ in $n$-SS patients compared to controls, which was less pronounced than the comparison between SS and controls $(p=0.0008)$. Th17 cells were decreased in SS compared to controls $(\mathrm{p}=0.0005)$, but not in-SS patients. Compared with controls, both $n-S S$ and SS patients presented decreased absolute count $(p=0.0001$ and $p<0,0001$, respectively) of CXCR5 ${ }^{+}$Tfh cells, with no differences between n-SS and SS patients. However, higher levels of IL2 $1^{+} \mathrm{CD} 4 \mathrm{~T}$ cells and Tfh1 cells were found comparing SS patients with both controls ( $p=0,0209$ and $p=0,0092$ respectively) and $n$-SS patients ( $p=0,0051$ and 0,0028 respectively).

Absolute counts of memory, unswitched and switched memory cells in n-SS patients present intermediate levels between controls with significantly higher levels, and SS patients with significantly lower levels. Accordingly, using the Bm1-5 classification, we have found decreased Bm1 ( $\mathrm{p}=0.004)$, eBm5 (Abs, $p=0.0273$ ) and Bm5 cells (Abs, $p=0.0444)$ in $n-S S$ patients compared to controls. Though not significant, there was an increase in eBm5 (Abs, $\mathrm{p}=0.063$ ) and Bm5 cells (Abs, $\mathrm{p}=0.05$ ) in $\mathrm{n}$-SS compared to SS patients. Again, CD24 ${ }^{+} \mathrm{CD} 27^{+}$ Bregs were also decreased in n-SS patients compared to controls $(p=0.036)$, but increased in $n-S S$ compared to SS patients $(p=0,0007)$.

Conclusions: Our data showed that $n$-SS patients present immune disregulation, represented by alterations in the B cell compartment but also in Tfh subset, known to modulate the humoral immune response. Although less pronounced, these modifications resemble the ones found in SS patients. Wether n-SS is a stage in the evolution to SS remains to be clarified. The identification of a characteristic disregulation of the immune system in n-SS could be usefull for diagnostic and prognostic purposes.

Disclosure of Interest: None declared

DOI: 10.1136/annrheumdis-2017-eular.6367

\section{AB0014 DIFFERENTIAL EFFECTS OF A SINGLE DOSE OF CYCLOPHOSPHAMIDE ON CIRCULATING CELL SUBPOPULATIONS IN PATIENTS WITH VASCULITIDES AND AUTOIMMUNE SYSTEMIC DISEASES}

G. Gabcova ${ }^{1}$, Z. Mikulkova ${ }^{1}$, M. Skacelova $^{2}$, A. Petrackova ${ }^{1}$, A. Smrzova ${ }^{2}$, M. Schubertova ${ }^{2}$, F. Mrazek ${ }^{3}$, P. Horak ${ }^{2}$, E. Kriegova ${ }^{1} .{ }^{1}$ Department of Immunology, Faculty of Medicine and Dentistry, Palacky University, Olomouc; ${ }^{2}$ Department of Internal Medicine III - Nephrology, Rheumatology and Endocrinology, Faculty of Medicine and Dentistry, Palacky University Olomouc, University Hospital, Olomouc; ${ }^{3}$ Department of Immunology, University Hospital Olomouc, Olomouc, Czech Republic

Background: High-dose cyclophosphamide (CFA) is used for treatment of patients with severe manifestations of systemic autoimmune diseases. The knowledge about the effect of CFA on circulating cell populations in these disease is still limited.

Objectives: To identify the effect of a single dose of CFA on circulating cell populations in patients with vasculitides and autoimmune systemic diseases.

Methods: We immunophenotyped T lymphocytes (CD3, CD4, CD8, CD25, CD127, HLA-DR), B lymphocytes (CD19, CD20, CD27), NK cells (CD3, CD16 CD56, CD69), neutrophils (CD15, CD11b, CD16, CD54, CD62L, CD64), and monocytes (CD11b, CD14, CD16, CD64, HLA-DR) using 6-color cytometer BD FACSCanto II (Becton Dickinson) in peripheral blood of patients with vasculitides $(n=6)$ and systemic disorders of connective tissue $(n=6)$. From each patient, we obtained paired samples before and one month after a single CFA dose. Statistical tests were performed using GraphPad Prism (GraphPad Software, Inc). P-value $<0.05$ was considered as significant.

Results: Single dose of CFA resulted in increase of percentage of CD8+ $\mathrm{T}$ lymphocytes $(P=0.002)$, leading to marked decrease of $\mathrm{CD} 4+/ \mathrm{CD} 8+$ ratio $(P=0.009)$. Except three patients, overall percentage of neutrophils decreased after the treatment $(P=0.01)$. Although CFA pulse did not influence the percentage of NK cells, the percentage of NK cells carrying stimulatory receptor CD69 increased after CFA $(P=0.037)$. Similarly, CFA enhanced the percentage of co-stimulatory molecule CD27 on B lymphocytes $(P=0.048)$. Among subsets of monocytes, CFA treatment increased percentage of CD14-CD16+ monocytes $(P=0.006)$ and increased expression (MFI) of FC fragment CD64 $(P=0.02)$. Moreover, increase of HLA-DR was observed on CD14-CD16+ monocytes $(P=0.037)$. The investigations whether the changes in immune cell subpopulations in treated patients have prognostic potential are ongoing.

Conclusions: Single dose of CFA resulted in increase of CD8+ T lymphocytes, activation of NK, B lymphocytes and monocytes as well as in some patients decrease in neutrophil counts. Further investigation of selected markers may lead to identification of new prognostic markers and predict the effectiveness of the treatment.

Acknowledgements: Grant support: MZ CR VES15-28659A, IGA_LF_2017_009 Disclosure of Interest: None declared

DOI: 10.1136/annrheumdis-2017-eular.4663

\section{AB0015 CAPTURE OF IGA IMMUNE COMPLEXES AND ENRICHMENT IN IGA IG GENE EXPRESSION BOTH SUGGEST A ROLE FOR FCRL4+ B CELLS IN THE LINK BETWEEN MUCOSAL AND JOINT INFLAMMATION}

J. Cameron $^{1}$, E. Clay ${ }^{1}$, K. Amara ${ }^{2}$, N. Sippl ${ }^{2}$, A. Filer ${ }^{1}$, K. Raza ${ }^{1}$,

V. Malmström ${ }^{2}$, D. Scheel-Toellner ${ }^{1}$. ${ }^{1}$ Rheumatology Research Group, RACE AR UK Centre of Excellence in RA Pathogenesis, Institute of Inflammation and Ageing, University of Birmingham, Birmingham, United Kingdom; ${ }^{2}$ Rheumatology Unit, Department of Medicine, Karolinska Universitetssjukhuset, Stockholm, Sweden

Background: Increasing evidence points to the autoimmune process of rheumatoid arthritis (RA) originating at mucosal surfaces. Previous work from our group described a subset of $B$ cells in the synovium and synovial fluid (SF) of RA patients which can be distinguished by their expression of the Fc-like receptor 4 (FcRL4) and elevated expression of RANKL, indicating a unique pathogenic function ${ }^{1,2}$. B cells expressing FCRL4 were originally described as a distinct memory B cell subset in human tonsils where they accumulate in the epithelium ${ }^{3}$. We have recently shown that they are enriched in cells recognizing citrullinated autoantigens (Amara, K. et al. under revision). Recent in vitro work suggested that FCRL4 is a low affinity receptor for aggregated $\lg \mathrm{A}^{4}$

Objectives: 1) To investigate the interaction of RA synovial fluid FCRL4+B cells with $\lg A$

2) To examine the distribution of Ig classes by flow cytometry and PCR

Methods: SF mononuclear cells were isolated, labelled for FCRL4, $\lg A$ and CD19 and analysed by flow cytometry. In experiments identifying IgA B cell receptors, SF mononuclear cells were briefly incubated in an acidic buffer to remove surface receptor bound antibodies before staining. Heat-aggregated purified human $\lg \mathrm{A}$ 